\title{
The Role of Cultural Leaders in the Transmission of Preferences
}

\author{
Thierry Verdier* $\quad$ Yves Zenou ${ }^{\dagger}$
}

August 25, 2015

\begin{abstract}
This paper studies the population dynamics of preference traits in a model of intergenerational cultural transmission with cultural leaders who compete for oblique socialization. We show that by adding this new chanel in the transmission of preferences, i.e. cultural leaders, in steady-state, there cannot be an equilibrium with total assimilation or total integration of the population.
\end{abstract}

JEL Classification Numbers: J13, J15, Z10.

Keywords: Socialization, cultural leaders, assimilation, integration.

*PSE-ENPC and CEPR. Email: thierry.verdier@ens.fr.

${ }^{\dagger}$ University of Stockholm, IFN and CEPR. Email: yves.zenou@ne.su.se. 


\section{Introduction}

An intense political and intellectual debate is taking place in Europe around migration issues, mainly focusing on the perceived costs and benefits of cultural diversity. While cultural diversity is generally seen a desirable societal trait, the persistence of ethnic identities on the part of minorities is often perceived by natives as a threat or as a source of potential problems (see e.g. Alba, 2005). The media often puts forward the role of community leaders in immigrant communities as an important aspect of the integration of these immigrants. Despite this, the notion of a leader who influences the assimilation or integration of ethnic minorities has not been very much studied in economics.

The aim of this paper is to tackle this issue by incorporating cultural leaders in a standard transmission model a la Bisin and Verdier. ${ }^{1}$ We consider a model where there are two traits, say "religious" and "secular". There is a social (vertical) transmission from older to younger generations. People (of all cultures) want to raise their children in their own traditions or culture. Religious parents want their children to be religious while secular parents want their offsprings to be secular (as in Patacchini and Zenou, 2015). There are also two types of horizontal transmission. First, there are passive role models with whom naive children can be randomly matched. Second and more importantly, there are also active role models such as cultural leaders (Imams, for example) who do interact strategically in the socialization process and spend resources influencing cultural evolution in a way that favors their interests. We characterize the steady state equilibrium and show that it is unique and always interior. Contrary to the standard Bisin-Verdier model, we shows that, by introducing cultural leaders, in steady-state, there cannot be an equilibrium with total assimilation or total integration of the population. We also show under which condition the majority of the population will be assimilated or integrated to the majority trait.

There are very few papers analyzing the role of leaders in a cultural-transmission model. Two notable exceptions are Hauk and Mueller (2015) and Prummer and Siedlarek (2015). In a different framework than ours, Prummer and Siedlarek (2015) explain the persistent differences in the cultural traits of immigrant groups with the presence of community leaders while Hauk and Mueller (2015) discuss the role of cultural leaders in the clash of cultures.

\footnotetext{
${ }^{1}$ See Bisin and Verdier $(2000,2001)$ and the survey by Bisin and Verdier (2011).
} 


\section{Model}

In this section, we present a simple model of cultural evolution in a two-cultural trait population of individuals. We build on the model of cultural transmission of Bisin and Verdier $(2000,2001)$ in which vertical socialization inside the family interacts with oblique socialization outside the family. Contrary to Bisin and Verdier (2001), we assume that cultural influence outside the family depends on two types of role models. First, there are passive role models with whom naive children can be randomly matched. Second and more importantly, there are also active role models such as cultural leaders who do interact strategically in the socialization process but who spend resources influencing cultural evolution in a way that favors their interests. To summarize, our mechanisms of cultural transmission assume the relevance of vertical transmission (from parents to children), horizontal transmission (measured by peer effects, which we refer to as the passive role models) and oblique socialization through other agents such as authority figures (here active role models such as cultural leaders).

To be more specific, suppose there are two possible types of cultural traits in the population denoted by $a$ and $b$ (they could refer to religious and non-religious, etc.). The fraction of individuals with trait $i \in\{a, b\}$ is denoted by $q^{i}$. Families are composed of one parent and a child, and hence reproduction is a-sexual. All children are born without defined preferences or cultural traits and are first exposed to their parent's trait. Direct vertical socialization to the parent's trait, say $i$, occurs with probability $d^{i}$. If a child from a family with trait $i$ is not directly socialized, which occurs with probability $1-d^{i}$, she is then subject to outside socialization, which depends on two components. With probability $1-\gamma$, the child is matched to a passive role model randomly chosen in the society. In such case, she adopts trait $i$ with probability $q^{i}$ and trait $j \neq i$ with probability $q^{j}=1-q^{i}$.

With probability $\gamma$, the child gets exposed to a cultural leader of trait $i$ or $j \neq i$. She then becomes socialized to trait $i$ with probability $\pi^{i}\left(\phi^{i}, \phi^{j}\right)$ and with probability $\pi^{j}\left(\phi^{j}, \phi^{i}\right)=$ $1-\pi^{i}\left(\phi^{i}, \phi^{j}\right)$ to the other trait $j \neq i$, where $\phi^{i}$ and $\phi^{j}$ are the socialization efforts of the leaders of types $i$ and $j$, respectively. We assume that there are two cultural leaders, one of type $i$ and one of type $j \neq i$, that compete with each other to impose their trait. For example, consider the Muslim community and think of traits $i$ and $j$ as "religious" and "secular" traits. Then, the cultural leader of type $i$, the religious leader, for example the Imam, will compete against the cultural leader of type $j$, a prominent secular Muslim leader, to impose his trait. For simplicity, we take a specific well-know contest function (Hirshleifer, 
(1989; Skaperdas, 1996; Konrad, 2009) given by:

$$
\pi^{i}\left(\phi^{i}, \phi^{j}\right)=\frac{\phi^{i}}{\phi^{i}+\phi^{j}}
$$

This formulation captures the fact that cultural leaders are in competition with each other for cultural transmission. The probabilities $\pi^{i}\left(\phi^{i}, \phi^{j}\right)$ can then be interpreted as cultural contest functions that satisfy the usual properties while $\gamma$ reflects the relative importance of cultural leaders in the transmission process as compared to the rest of society.

Denote as $P^{i j}$ denote the probability that a child from a family with trait $i$ is socialized to trait $j$. By the Law of Large Numbers, $P^{i j}$ also denotes the fraction of children with a type $i$ parent who have preferences of type $j$. The socialization mechanism described above can then be characterized by the following transition probabilities, for all $i, j \in\{a, b\}$ :

$$
\begin{gathered}
P^{i i}\left(d^{i}, \phi^{i}, \phi^{j}, \gamma, q^{i}\right)=d^{i}+\left(1-d^{i}\right)\left[\gamma \pi^{i}\left(\phi^{i}, \phi^{j}\right)+(1-\gamma) q^{i}\right] \\
P^{i j}\left(d^{i}, \phi^{i}, \phi^{j}, \gamma, q^{i}\right)=\left(1-d^{i}\right)\left[\gamma \pi^{j}\left(\phi^{j}, \phi^{i}\right)+(1-\gamma)\left(1-q^{i}\right)\right]
\end{gathered}
$$

The dynamics of $q^{i}$, the fraction of the population with trait $i \in\{a, b\}$, can then be written as:

$$
q_{t+1}^{i}=q_{t}^{i} P^{i i}\left(d^{i}, \phi^{i}, \phi^{j}, \gamma, q^{i}\right)+\left(1-q_{t}^{i}\right) P^{j i}\left(d^{j}, \phi^{j}, \phi^{i}, \gamma, q^{i}\right)
$$

Using the notation $\pi^{i} \equiv \pi^{i}\left(\phi^{i}, \phi^{j}\right)$ for $i \neq j$ and using (2) and (3), for $i \in\{a, b\}$, this equation can be written as:

$$
q_{t+1}^{i}=q_{t}^{i}\left[d^{i}+\left(1-d^{i}\right)\left(\gamma \pi^{i}+(1-\gamma) q_{t}^{i}\right)\right]+\left(1-q_{t}^{i}\right)\left(1-d^{j}\right)\left(\gamma \pi^{i}+(1-\gamma) q_{t}^{i}\right)
$$

where $\pi^{i} \equiv \pi^{i}\left(\phi^{i}, \phi^{j}\right)$ is given by (1). In this section, we assume that the family socialization rates $d^{i}$ for $i \in\{a, b\}$ are exogenous. In contrast, we consider that cultural leaders enjoy rents associated to the number of individuals who share their cultural traits in the population and therefore are ready to spend resources and efforts to affect the process of cultural evolution in society. Specifically, in each period $t$, the society is characterized by a population state with $q_{t}^{i}$ families having parents of type $i \in\{a, b\}$. After the socialization process described above, children inside these families acquire their own trait and become adults in period $t+1$ with cultural dynamics according to (4). Anticipating such cultural evolution for time $t+1$, leaders of type $i \in\{a, b\}$ play a contest influence game and simultaneously choose their socialization rates $\phi^{i}$ in order to maximize their objective function, given by:

$$
W^{i}\left(q_{t}^{i}, \phi^{i}, \phi^{j}\right)=R^{i}\left(q_{t+1}^{i}\right)-C\left(\phi^{i}\right)
$$


where $R^{i}\left(q_{t+1}^{i}\right)$ is the rent that a cultural leader of type $i$ expects to receive in period $t+1$. This rent is assumed to positively depend on the fraction $q_{t+1}^{i}$ of adult individuals that at time $t+1$ will have adopted the trait promoted by leader $i$. For simplicity, we assume that the rent is proportional to $q_{t+1}^{i}$ and takes the form: $R^{i}\left(q_{t+1}^{i}\right)=V^{i} q_{t+1}^{i}$, where $V^{i}$ is the marginal benefit that the cultural leader $i$ obtains from increasing her cultural influence. $C\left(\phi^{i}\right)$ is the resource cost for the leader to obtain a socialization rate of $\phi^{i}$. For simplicity, we also assume that it is also linear in $\phi^{i}$ so that $C\left(\phi^{i}\right)=c \phi^{i}$, where $c$ is the marginal cost of influence. Observe that we assume that, in each period $t$, cultural leaders are only one step forward-looking in the sense that they only care about the cultural evolution outcome of period $t+1$.

\section{Steady-state equilibrium}

At each period $t$, we can easily solve the Nash equilibrium in cultural influence of the two leaders. For that, one may first compute the best-response function of a leader of type $i$, given what the other leader is playing. For an interior solution, best-response function of a leader of type $i$ is given by:

$$
V^{i} \frac{\partial q_{t+1}^{i}}{\partial \phi^{i}}-c=0
$$

Using (4) and (1), this can be written as:

$$
V^{i} \frac{\gamma \phi^{j}}{\left(\phi^{i}+\phi^{j}\right)^{2}}\left[q_{t}^{i}\left(1-d^{i}\right)+\left(1-q_{t}^{i}\right)\left(1-d^{j}\right)\right]-c=0
$$

Solving this equation for $\phi$ leads to:

$$
\left(\phi^{i}\right)^{2} c+2 c \phi^{j} \phi^{i}+c\left(\phi^{j}\right)^{2}-V^{i} \gamma \phi^{j}\left[q_{t}^{i}\left(1-d^{i}\right)+\left(1-q_{t}^{i}\right)\left(1-d^{j}\right)\right]=0
$$

Solving this second-degree equation leads to a unique positive value of $\phi^{i}$ given by:

$$
\phi^{i}\left(\phi^{j}, q_{t}^{i}, d^{i}, V^{i}\right)=-\phi^{j}+\frac{\sqrt{c V^{i} \gamma \phi^{j}\left[q_{t}^{i}\left(1-d^{i}\right)+\left(1-q_{t}^{i}\right)\left(1-d^{j}\right)\right]}}{c}
$$

It follows from simple differentiation that the best-response function $\phi^{i}\left(\phi^{j}, q_{t}^{i}, d^{i}, V^{i}\right)$ is increasing in the marginal cultural rent value $V^{i}$, is decreasing in the marginal cost $c$ of socialization and is increasing in the weight $\gamma$ of cultural leaders in the cultural process. More interestingly, the best response function $\phi^{i}\left(\phi^{j}, q_{t}^{i}, d^{i}, V^{i}\right)$ is increasing in the frequency $q_{t}^{i}$ of group $i$ if and only if $d^{j}-d^{i} \geq 0$, that is when families of type $i$ are less successful 
in directly transmitting their trait than families of type $j$. Indeed, in such a case, an increase in $q_{t}^{i}$ increases the number of children in the population that can be influenced by cultural leaders. This makes the market of cultural influence larger, increasing therefore the profitability of socialization for both types of leaders.

As it is standard for contest best-reply functions, the dependence of $\phi^{i}\left(\phi^{j}, q_{t}^{i}, d^{i}, V^{i}\right)$ on $\phi^{j}$ is generally non-monotonic. This is what we obtain here. Indeed, at low levels of $\phi^{j}$, the leader socialization rates are strategic complements (i.e. $\phi^{i}\left(\phi^{j}, q_{t}^{i}, d^{i}, V^{i}\right)$ is increasing in $\phi^{j}$ ) while they may eventually become strategic substitutes for higher values of $\phi^{j}$ (i.e. $\phi^{i}\left(\phi^{j}, q_{t}^{i}, d^{i}, V^{i}\right)$ decreases in $\left.\phi^{j}\right)$.

Finally, notice that $\phi^{i}=\phi^{i}\left(\phi^{j}, q_{t}^{i}, d^{i}, V^{i}\right)$, the optimal socialization effort of a cultural leader of type $i$ depends on the socialization rates $d^{i}$ and $d^{j}$ of the families. As in Bisin and Verdier (2001), this reflects a form of cultural substituability, except that it is now related to the endogenous effort of socialization of the external role models (cultural leaders) rather than the parents.

If trait $i=a$ and trait $j=b$, then (5) can be written as:

$$
\begin{aligned}
\phi^{a} & =-\phi^{b}+\frac{\sqrt{c V^{a} \gamma \phi^{b}\left[q_{t}^{a}\left(1-d^{a}\right)+\left(1-q_{t}^{a}\right)\left(1-d^{b}\right)\right]}}{c} \\
\phi^{b} & =-\phi^{a}+\frac{\sqrt{c V^{b} \gamma \phi^{a}\left[q_{t}^{b}\left(1-d^{b}\right)+\left(1-q_{t}^{b}\right)\left(1-d^{a}\right)\right]}}{c}
\end{aligned}
$$

By combining these two equations and noticing that $q^{b}=1-q^{a}$, at the Nash equilibrium, we have:

$$
\frac{\phi^{a}}{\phi^{b}}=\frac{V^{a}}{V^{b}}
$$

Using (1), this implies that

$$
\pi^{a *}=1-\pi^{b *}=\frac{\phi^{a}}{\phi^{a}+\phi^{b}}=\frac{V^{a}}{V^{a}+V^{b}}
$$

This is a nice and neat result that shows that the probability of transmitting the trait $a$ for the leader of type $a$ is just equal to the relative effort of the leaders, which, in turn, is equal to the relative marginal benefit that the leader of type $a$ obtains from increasing her cultural influence.

Let us now study the evolution of the population with a certain trait. Since there are only two traits $a$ and $b$, then $q_{t}$ denotes the fraction of families with trait $a$ at time $t$ while 
$1-q_{t}$ denotes the fraction of families with trait $b$ at time $t$. This means that the cultural dynamics equation (4) can be written as:

$$
q_{t+1}=q_{t}\left[d^{a}+\left(1-d^{a}\right)\left(\gamma \pi^{a *}+(1-\gamma) q_{t}\right)\right]+\left(1-q_{t}\right)\left(1-d^{b}\right)\left[\gamma \pi^{a *}+(1-\gamma) q_{t}\right]
$$

which is equivalent to

$$
q_{t+1}-q_{t}=\left(1-d^{b}\right)\left(1-q_{t}\right)\left[\gamma \pi^{a *}+(1-\gamma) q_{t}\right]-\left(1-d^{a}\right) q_{t}\left[1-\gamma \pi^{a *}-(1-\gamma) q_{t}\right]
$$

By rearranging the terms in this equation, we obtain:

$$
q_{t+1}-q_{t}=q_{t}\left(1-q_{t}\right)(1-\gamma)\left(d^{a}-d^{b}\right)-\gamma\left[\left(1-d^{a}\right) q_{t}\left(1-\pi^{a *}\right)-\left(1-d^{b}\right) \pi^{a *}\left(1-q_{t}\right)\right]
$$

where $\pi^{a *}$ is given by (7).

We obtain the following result that characterizes the steady state cultural evolution:

Proposition 1 For any given initial condition $q(0)=q_{0}$, the cultural dynamics (8) converges to a unique interior steady state $\left.q^{*} \in\right] 0,1[$.

(i) If $d^{a}>d^{b}$, then the unique steady-state equilibrium is such that

$$
(1-\gamma)\left(V^{a}+V^{b}\right)\left(d^{a}-d^{b}\right)+2 \gamma\left[\left(V^{b} d^{a}-V^{a} d^{b}\right)+\left(V^{a}-V^{b}\right)\right] \gtreqless 0 \Leftrightarrow q^{*} \gtreqless 1 / 2
$$

(ii) If $d^{a}<d^{b}$, then the unique steady-state equilibrium is such that $0<q^{*}<1 / 2$.

Proof: See the Appendix.

The characterization of the equilibrium interior steady state is depicted in Figure 1 when $d^{a}>d^{b}$ and condition (9) holds with a positive sign, in Figure 2 when $d^{a}>d^{b}$ and condition (9) holds with a negative sign, and in Figure 3 when $d^{a}<d^{b}$.

\section{[Insert Figures 1, 2 and 3 here]}

Several observations are worth mentioning. First, note that $q^{*}=0$ or $q^{*}=1$ cannot be a steady-state equilibrium here while, in the standard Bisin-Verdier model, they are always equilibria and no interior equilibrium can exist. In other words, the cultural leader avoids 
the total assimilation of the population $\left(q^{*}=0\right)$ or its total integration $\left(q^{*}=1\right)$. Indeed, if $\gamma=0$, i.e. no leader, then the steady-state equilibrium is given by:

$$
q^{*}\left(1-q^{*}\right)\left(d^{a}-d^{b}\right)=0
$$

where, clearly, only corner solutions are steady-state equilibria. Second, if parents put the same effort in transmitting their traits, i.e. $d^{a}=d^{b}=d$, then the steady-state equilibrium with leaders (10) is given by:

$$
q^{*}=\pi^{a *}=\frac{\phi^{a}}{\phi^{a}+\phi^{b}}=\frac{V^{a}}{V^{a}+V^{b}}
$$

which implies that

$$
q^{*} \gtreqless \frac{1}{2} \Leftrightarrow \phi^{a} \gtreqless \phi^{b}
$$

In other words, if we shut down the vertical transmission of the parents, then the fraction of the population with trait $a$ will only depend on the socialization rates $\phi^{a}$ and $\phi^{b}$ chosen by the leaders. Finally, if there is only one leader of type $a$ (resp. type $b)$, i.e. $\phi^{b}=0\left(\phi^{a}=0\right)$, then $\pi^{a *}=1\left(\pi^{b *}=1\right)$, then $q^{*}=1$ if $d^{a}>d^{b}\left(q^{*}=0\right.$ if $\left.d^{b}>d^{a}\right)$. In other words, the difference in parents' efforts will determine the assimilation or integration of the population.

\section{Conclusion}

It is well-documented that cultural leaders have an important impact on the assimilation or integration of immigrants in Europe. For example, Imams have a key role in the integration of Muslims in Germany (Prummer and Siedlarek, 2015). To understand this issue, we develop a simple model where individuals can adopt two possible traits, say "religious" and "secular". There is one leader for each trait and each of them wants to "push" their own trait in the population. We show that, in equilibrium, there cannot be a complete assimilation $\left(q^{*}=0\right.$ so that all individuals are secular $)$ or integration $\left(q^{*}=1\right.$ so that all individuals are religious) of the population. We also show that if both parents put the same effort in transmitting their traits, then the fraction of the population being religious will only depend on the socialization rates chosen by the leaders.

\section{References}

[1] Alba R.D. (2005), "Bright vs. blurred boundaries: Second generation assimilation and exclusion in France, Germany and the United States," Ethnic and Racial Studies 28, $20-59$. 
[2] Bisin, A. and T. Verdier (2000), "Beyond the melting pot: Cultural transmission, marriage, and the evolution of ethnic and religious traits," Quarterly Journal of Economics 115, 955-988.

[3] Bisin, A. and T. Verdier (2001), "The economics of cultural transmission and the dynamics of preferences," Journal of Economic Theory 97, 298-319.

[4] Bisin, A. and T. Verdier (2011), "The economics of cultural transmission and socialization," In J. Benhabib, A. Bisin, and M.O. Jackson (Eds.), Handbook of Social Economics, Amsterdam: Elsevier Science, pp. 339-416.

[5] Hirshleifer, J. (1989), "Conflict and rent-seeking success functions: Ratio vs. difference models of relative success," Public Choice 63, 101-112.

[6] Hauk, E. and H. Mueller (2015), "Cultural leaders and the clash of civilizations," Journal of Conflict Resolution 59, 367-400.

[7] Konrad, K.A. (2009), Strategy and Dynamics in Contests, New York: Oxford University Press.

[8] Patacchini, E. and Y. Zenou (2014), "Social networks and parental behavior in the intergenerational transmission of religion," CEPR Discussion Paper No. 8443.

[9] Prummer, A. and J.-P. Siedlarek (2015), "Community leaders and the preservation of cultural traits," Unpublished manuscript, University of Mannheim.

[10] Skaperdas, S. (1996), "Contest success functions," Economic Theory 7, 283-290. 


\section{Appendix}

Proof of Proposition 1: To calculate the steady-state value of $q^{*}$, we impose that: $q_{t+1}-q_{t}=0$ in (8) and obtain:

$$
q^{*}\left(1-q^{*}\right)\left(\frac{1-\gamma}{\gamma}\right)\left(d^{a}-d^{b}\right)=\left(1-d^{a}\right) q^{*}\left(1-\pi^{a *}\right)-\left(1-d^{b}\right) \pi^{a *}\left(1-q^{*}\right)
$$

Denote

$$
\Phi(q) \equiv q(1-q)\left(\frac{1-\gamma}{\gamma}\right)\left(d^{a}-d^{b}\right)
$$

and

$$
\Omega(q) \equiv\left(1-d^{a}\right) q\left(1-\pi^{a *}\right)-\left(1-d^{b}\right) \pi^{a *}(1-q)
$$

First, observe that $\Phi(0)=\Phi(1)=0$ and, for $q \in] 0,1\left[, \Phi(q) \gtrless 0 \Leftrightarrow d^{a} \gtrless d^{b}\right.$. As a result, if $d^{a}>d^{b}$, then $\Phi(q)$ can be represented in the positive orthant as a concave function with a maximum at $q=1 / 2$ (Figures 1 and 2). On the contrary, if $d^{a}<d^{b}, \Phi(q)$ can be represented in the negative orthant as a convex function with a minimum at $q=1 / 2$ (Figure 3).

Second, $\Omega(0)<0$, and, using $(7), \Omega\left(q_{\Omega}\right)=0$, where

$$
q_{\Omega}=\frac{\left(1-d^{b}\right) V^{a}}{\left(1-d^{b}\right) V^{a}+\left(1-d^{a}\right) V^{b}}
$$

Thus, for $q \in[0,1], \Phi(q) \lesseqgtr 0 \Leftrightarrow q \gtreqless q_{\Omega}$. As a result, $\Omega(q)$ can be represented by an upward sloping line as in Figures 1 and 2.

(i) Case: $d^{a}>d^{b}$. Since $0<q_{\Omega}<1$, then there is a unique steady-state $q^{*}$ such that $0<q^{*}<1$ (Figures 1 and 2). Moreover, it is easily verified that

$$
\Phi(1 / 2) \gtreqless \Omega(1 / 2) \Leftrightarrow q^{*} \gtreqless 1 / 2
$$

This is equivalent to:

$$
(1-\gamma)\left(V^{a}+V^{b}\right)\left(d^{a}-d^{b}\right)+2 \gamma\left[\left(V^{b} d^{a}-V^{a} d^{b}\right)+\left(V^{a}-V^{b}\right)\right] \gtreqless 0
$$

which is the condition given in the Proposition.

(ii) Case: $d^{a}<d^{b}$. Since $0<q_{\Omega}<1$, then there is a unique steady-state $q^{*}$ such that $0<q^{*}<1$ (Figure 3). It is then easily verified that this steady-state equilibrium $q^{*}$ is such that $1 / 2<q^{*}<1$. Figure 3 describes this equilibrium. 


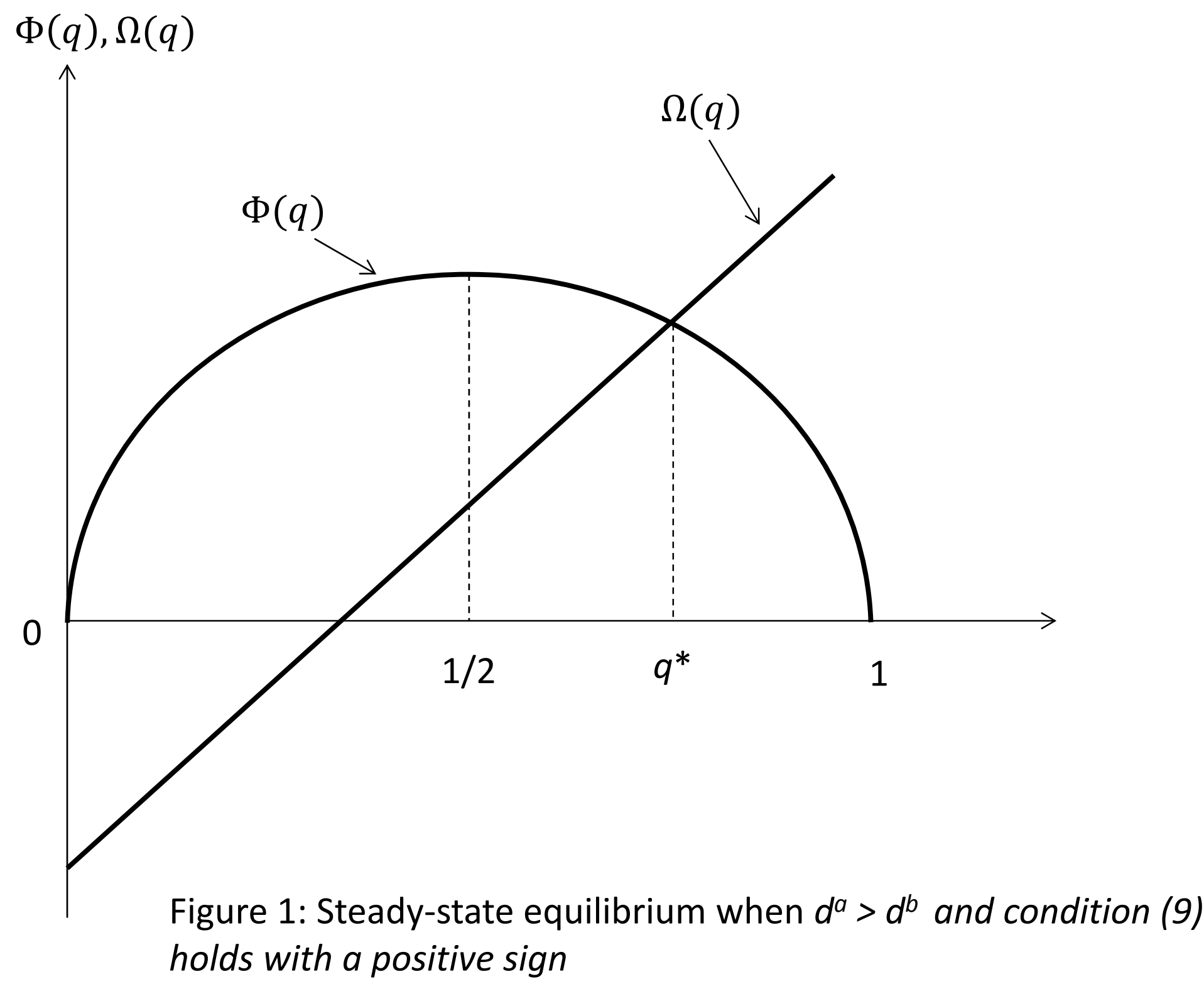




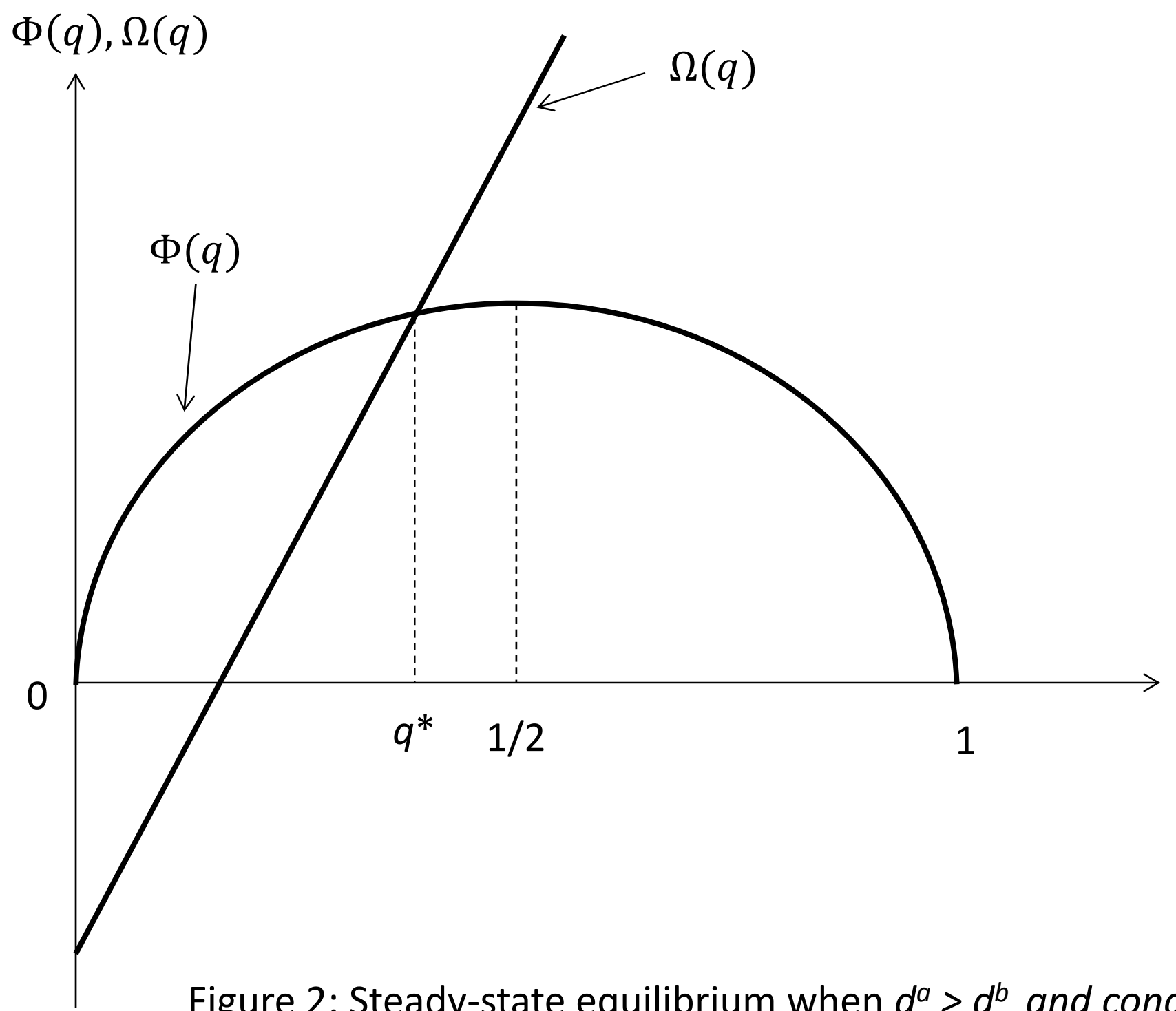

Figure 2: Steady-state equilibrium when $d^{a}>d^{b}$ and condition (9) holds with a negative sign 


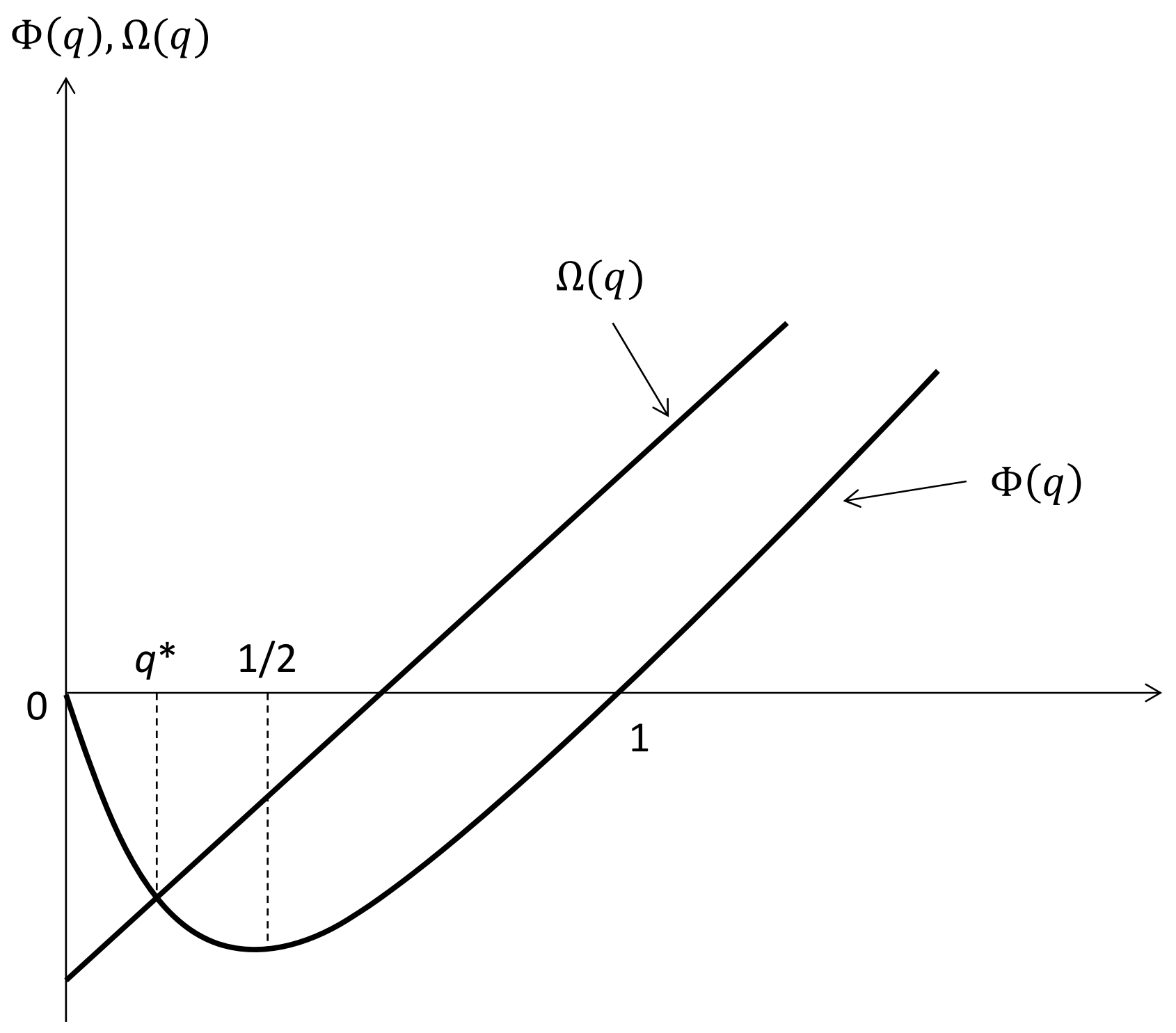

Figure 3: Steady-state equilibrium when $d^{a}<d^{b}$ 